\title{
Robust Slope Region for Wideband CDMA with Multiple Antennas \\ Invited paper
}

\author{
Tarik Muharemović \\ tarik@rice.edu
}

\author{
Behnaam Aazhang \\ aaz@rice.edu
}

Abstract - We analyze a low power, wideband CDMA system with multiple antennas. The joint "slope region" of transmission rates at minimum energy per bit - is derived as a function of proportion between vanishing rates for multiple users. Vertices of the slope region may be achieved by a matched filter linear interface, followed by successive interference cancellation. We introduce and evaluate "robust slope region," the largest region which is inside every other slope region and is therefore independent from the relative proportion between transmission rates. Furthermore, we find the "robust slope," maximum slope which can always be guaranteed to all users.

\section{INTRODUCTION}

A new set of analysis tools was provided in [1], which allows us to investigate the tradeoff of spectral efficiency and energy efficiency for various wideband systems. Subsequently, two user multiple access channel was considered in [2,3], and a fundamental notion of "slope region" was introduced as a function of unevenness between transmission rates. Given that the two rates vanish in a certain proportion $\theta$, there is a set of admissible pairs of slopes of rates at minimum energy per bit.

A brief reflection on the minimum energy per bit in both single and multiuser communication mode [1] establishes that it depends only on the channel statistics. It is independent from relative proportion between users rates or powers.

One can think of transmission rate as either a function of power, or as a function of energy per bit. The later one "starts off" at minimum energy per bit $E b N 0_{\text {min }}$, and we are interested in its initial slope. In single user communication mode, this slope depends only on the channel statistics [1].

(Dis)proportion between user powers and/or rates factors into multiple access slope region [2]. Only if users are sufficiently power unbalanced, individual single user slopes become simultaneously achievable. This may be thought of as an effective way of user separation - by powers.

Here we evaluate portion of the slope region which is independent or the relative proportion between the rates. It lies inside every other slope region and that's why perhaps the best name for it is the robust slope region. Furthermore, we evaluate the symmetric robust slope, as the slope which can always be granted to all users. In a scenario where relative balance between users transmission rates or powers is not fixed, robust slope gauges the worst case bandwidth requirements. This makes it an important parameter in wideband system design. We proceed in full generality, assuming multiple users, and incorporating features such as multiple antennas and spreading codes.

Finally, we demonstrate how vertices of the slope region may be achieved by a matched filter linear interface, provided that we employ successive interference cancellation. We also evaluate the bandwidth penalty due to orthogonalization between users for a many user system.

\section{PRELIMINARIES}

We consider $K$-user multiple access channel model, where each user has $N_{k}$ transmit antennas and power $P_{k}$. There are a total of $M$ receive antennas. Symbols of each user are modulated by a column spreading sequence $s_{k}$ of length $L$ and unit energy. User separation is therefore twofold: spatial and temporal. Still, inherent differences between the two types of separation justify the "disjoint approach". Spatial separation could be statistical, while temporal may be deterministic by design.

Wireless system designer can by no means control the fading environment of many users. They may be located in various scattering environments, having different transmit correlation. On the other hand, some of them may be significantly close so that their channels are correlated due to the same propagation environment. Wideband analysis helps us quantify the achievable set of transmission rates slopes at the minimum energies per bit for all above mentioned scenarios. Let columns of the received $M \times L$ matrix $y$ represent consecutive chip intervals, then

$$
y=\sum_{1 \leq k \leq K} H_{k} x_{k} s_{k}^{T}+\eta .
$$

Complex additive white Gaussian noise with unit variance is represented by the matrix $\eta$. We read the received matrix $y$ columnwize by applying the "stack" operator $\cup[7]$

$$
y^{\cup}=\sum_{1 \leq k \leq K}\left(s_{k} \otimes H_{k}\right) x_{k}+\eta^{\cup} .
$$

Here, $\otimes$ denotes the Kronecker matrix product. We constrain all users inputs to be i.i.d, with equal power allocation along transmit antennas. Following are important matrix identities [7] used extensively throughout the analysis

$$
\begin{aligned}
\left(s_{k} \otimes H_{k}\right)\left(s_{k} \otimes H_{k}\right)^{\dagger} & =\left(s_{k} s_{k}^{\dagger}\right) \otimes\left(H_{k} H_{k}^{\dagger}\right) \\
\operatorname{tr}(B \otimes \hat{B}) & =\operatorname{tr}(B) \operatorname{tr}(\hat{B}) \\
\operatorname{tr}(B \hat{B}) & =\operatorname{tr}(\hat{B} B) \\
\operatorname{Etr}(B) & =\operatorname{tr} E(B) .
\end{aligned}
$$

We state the multiple access capacity region

$$
\begin{gathered}
\left(R_{1}, R_{2}, \ldots, R_{K}\right): \forall A \subseteq\{1,2, \ldots, K\} \\
\sum_{k \in A} R_{k} \leq E \log \left|I+\sum_{k \in A}\left(s_{k} s_{k}^{\dagger}\right) \otimes\left(H_{k} H_{k}^{\dagger}\right) \frac{P_{k}}{N_{k}}\right| .
\end{gathered}
$$


In order to perform wideband analysis we let all users rates approach zero. However, we maintain relative proportion between the rates [2]. For that purpose we normalize as follows: let the rates $R_{k}$ vanish in proportion, so that

$$
\forall A \subseteq\{1,2, \ldots, K\}, \quad \frac{R_{k}}{\sum_{k \in A} R_{k}}=\theta_{k}(A) .
$$

First and second order behavior of transmission rate of $k-$ th user as a function of signal to noise ratio $R_{k}\left(P_{k}\right)$ specify minimum energy per bit $E b N 0_{\min }(k)$, and the initial slope $\mathcal{S}_{k}$ of transmission rate as a function of energy per bit [1]. That is,

$$
\begin{gathered}
E b N 0_{\min }(k)=\frac{\log _{e} 2}{\dot{R}_{k}(0)} \\
\mathcal{S}_{k}=2 \frac{\left[\dot{R}_{k}(0)\right]^{2}}{-\ddot{R}_{k}(0)} .
\end{gathered}
$$

Here, $\dot{R}_{k}(0)$ and $\ddot{R}_{k}(0)$ are first and second derivative of transmission rate as a function of $P_{k}$, evaluated at zero. We also need following identities as stated in [1]

$$
\begin{gathered}
\left.\frac{d}{d P} \log |I+B P|\right|_{P=0}=\operatorname{tr}(B) \\
\left.\frac{d^{2}}{d P^{2}} \log |I+B P|\right|_{P=0}=-\operatorname{tr}\left(B^{2}\right)
\end{gathered}
$$

We first evaluate minimum energy per bit and the slope region for our channel model. Proofs are mostly outlined and without tedious details.

\section{The SLOPE REGION}

In single user communication mode minimum energy per bit may be found as

$$
\begin{aligned}
\lim _{P_{k} \rightarrow 0} \frac{R_{k}}{P_{k}} & =\dot{R}_{k}(0) \\
& =\frac{1}{N_{k}} \operatorname{Etr}\left[\left(s_{k} s_{k}^{\dagger}\right) \otimes\left(H_{k} H_{k}^{\dagger}\right)\right] \\
& =\frac{1}{N_{k}} \operatorname{Etr}\left(H_{k} H_{k}^{\dagger}\right) \\
& \equiv G_{k} .
\end{aligned}
$$

We have implicitly defined the channel gain $G_{k}$, as in [1]. Transmission rate $R$ is measured in nats for convenience. We see that the minimum energy per nat is just the reciprocal of the above quantity. It follows that the minimum energy per bit is given as

$$
E b N 0_{\min }(k)=\frac{\log _{e} 2}{G_{k}} .
$$

In a multiple access setting, this quantity may be achieved even by a matched filter detector [1]. Since presence of interference does not help, we conclude that even the optimal detector will attain this energy per bit.

When evaluating equations which define the "slope region," we begin from their counterparts from the "rate region" (7). Without loss of generality assume that $A$ encompasses all users and suppress that variable. We will evaluate the impact of the "last" equation from the rate region (7), assuming that it holds with equality. Define the total power seen by the receiver,

$$
P=\sum_{1 \leq k \leq K} G_{k} P_{k}
$$

Equations (13), (8) and (18) imply that in the limit

$$
P_{k}=\frac{R_{k}}{G_{k}}=\frac{\theta_{k} \sum_{k^{\prime}=1}^{K} R_{k^{\prime}}}{G_{k}}=\frac{\theta_{k}}{G_{k}} P .
$$

In order to evaluate first and second order behavior of $R_{k}$, as $P_{k}$ approaches zero, we investigate the behavior of $R_{k}$ as $P$ approaches zero. Chain rule for differentiation establishes the relationship between the two quantities. We begin by expressing $R_{1}$ as a convex combination of $K$ vertices from the rate region. Let $\sum_{i=1}^{K} \alpha_{i}=1$, then

$$
\begin{aligned}
R_{1}=\sum_{i=1}^{K} \alpha_{i} & {\left[E \log \left|I+\sum_{k=1}^{i}\left(s_{k} s_{k}^{\dagger}\right) \otimes\left(H_{k} H_{k}^{\dagger}\right) \frac{\theta_{k}}{N_{k} G_{k}} P\right|\right.} \\
& \left.-E \log \left|I+\sum_{k=2}^{i}\left(s_{k} s_{k}^{\dagger}\right) \otimes\left(H_{k} H_{k}^{\dagger}\right) \frac{\theta_{k}}{N_{k} G_{k}} P\right|\right] .
\end{aligned}
$$

Similar equations hold for $R_{j}, 1 \leq j \leq K$, only with indices (but not the boundary terms in the summation) shifted by $j-1$, modulo $K$. For example, $i$ becomes $i \oplus j \ominus 1$, and $k$ becomes $k \oplus j \ominus 1$. Furthermore, if we were to add all those equations we would obtain the "last" constraint from the rate region (7). The equation that needs verification is

$$
\begin{aligned}
\sum_{j=1}^{K} \sum_{i=1}^{K} \alpha_{i \oplus j \ominus 1} & {\left[\log \left|I+\sum_{k=1}^{i} B_{k \oplus j \ominus 1}\right|\right.} \\
& \left.-\log \left|I+\sum_{k=2}^{i} B_{k \oplus j \ominus 1}\right|\right] \\
& =\log \left|I+\sum_{s=1}^{K} B_{s}\right| .
\end{aligned}
$$

We can verify this using $i \oplus j \ominus 1=l$, and $i-k=s$ change of variable. Terms multiplied with each $\alpha_{l}$ will form a telescoping summation. Hence, the set of rates is truly a convex combination of the vertices. We can therefore proceed with calculation since we made sure that we gauged the "last" constraint. We evaluate the first order behavior of the expression for $R_{1}$ using equation (11). Since Etr is linear, the two inner summations collapse leaving only the term $k=1$. We reaffirm that

$$
\dot{R}_{1}(0)=G_{1}
$$

Second order behavior is also necessary to find the slope region. It may be evaluated using (12), and it is much more complicated,

$$
\begin{aligned}
-\ddot{R}_{1}(0) & =\frac{\operatorname{Etr}\left(H_{1} H_{1}^{\dagger}\right)^{2}}{N_{1}^{2}}+ \\
& +2 \frac{G_{1}^{2}}{M} \sum_{i=1}^{K} \alpha_{i} \sum_{k=2}^{i}|\rho(1, k)|^{2} \delta(1, k)\left(\frac{\theta_{k}}{\theta_{1}}\right) .
\end{aligned}
$$


The last equality follows after much tedious calculation and it is explained as follows. $\rho(k, l)$ is correlation between spreading sequences for users $k$ and $l$. "Dependence" between matrix channels for users $k$ and $l$ is denoted as $\delta(l, k)$, and is defined as

$$
\delta(k, l) \equiv M \frac{\operatorname{Etr}\left[H_{k} H_{k}^{\dagger} H_{l} H_{l}^{\dagger}\right]}{\operatorname{Etr}\left[H_{k} H_{k}^{\dagger}\right] \operatorname{Etr}\left[H_{l} H_{l}^{\dagger}\right]} .
$$

For purposes of this discussion we will name this variable $\delta$ factor. We comment on it further down. Using (10), and normalizing the slope per receiver antenna per chip, which is the total number of receive dimensions we arrive at

$$
\begin{aligned}
\frac{\theta_{1}^{2}}{\mathcal{S}_{1}} & =\frac{\theta_{1}^{2} L M}{2}\left(\frac{-\ddot{R}_{k}(0)}{\left[\dot{R}_{k}(0)\right]^{2}}\right) \\
& =\frac{L \theta_{1}^{2}}{\mathcal{S}_{1}\left(H_{1}\right)}+L \sum_{i=1}^{K} \alpha_{i} \sum_{k=2}^{i}|\rho(1, k)|^{2} \delta(k, l) \theta_{1} \theta_{k} .
\end{aligned}
$$

Following [1] we defined the slope in a single user communication mode, for user $k$ as

$$
\mathcal{S}_{k}\left(H_{k}\right)=\frac{2}{M} \frac{t^{2} E\left[H_{k} H_{k}^{\dagger}\right]}{\operatorname{tr} E\left[\left(H_{k} H_{k}^{\dagger}\right)^{2}\right]}=\frac{2}{\delta(k, k)} .
$$

Again, expressions similar to (25) hold for $\mathcal{S}_{l}, 1 \leq l \leq K$, only with indices (but not the boundary terms in the summation) shifted by $j-1$, modulo $K$. Adding all those equations we obtain the "last" constraint pertinent to the slope region. In general, the set of equation specifying the region is as follows

Theorem 1 Let the rates $R_{k}$ vanish in proportion, so that $\forall A \subseteq\{1,2, \ldots, K\}$ we have

$$
\frac{R_{k}}{\sum_{k \in A} R_{k}}=\theta_{k}(A)
$$

Furthermore, let $\Phi_{A}$ denote the matrix of square correlations between spreading sequences for users within $A$, and let $\Delta_{A}$ denote the matrix of $\delta$ factors. The optimum multiple access slope region admits surprisingly simple interpretation

$$
\begin{gathered}
\left(\mathcal{S}_{1}, \mathcal{S}_{2}, \ldots, \mathcal{S}_{K}\right): \forall A \subseteq\{1,2, \ldots, K\} \\
\sum_{k \in A} \frac{\theta_{k}^{2}(A)}{\mathcal{S}_{k}} \geq \frac{L}{2} \theta(A)^{T}\left[\Phi_{A} \odot \Delta_{A}\right] \theta(A) .
\end{gathered}
$$

Here $\odot$ denotes Hadamard or Schur ${ }^{1}$ matrix product, which is computed "index by index." Essentially, slopes have to be small enough so that the term on the left dominates the quadratic on the right.

If $\mathbb{S}_{A}$ is a matrix of spreading codes, note that total square correlations may be expressed as

$$
\Phi_{A}=\left(\mathbb{S}_{A}^{\prime} \mathbb{S}_{A}\right) \odot\left(\mathbb{S}_{A}^{\prime} \mathbb{S}_{A}\right) .
$$

All entries of $\Delta_{A}$ are greater than 1 , which could be shown using the fact that $E \operatorname{tr}[B \hat{B}]$ defines a valid inner product, as well as $\frac{1}{M} \operatorname{Etr}[B \hat{B}]$. As a simple case of correlated channel

\footnotetext{
${ }^{1}$ In MATLAB notation .*
}

model, we can express correlation between coefficients in the matrix channel into transmit correlation $C_{k}$ and receive correlation $\Gamma$,

$$
H_{k}=\sqrt{\Gamma} \hat{H}_{k} \sqrt{C}_{k} .
$$

Here, $\hat{H}_{k}$ is i.i.d. If users $k$ and $l$ have independent channels (between each other), using (23), we can verify that $\delta(k, l)$ is independent of the transmit correlation,

$$
\delta(k, l)=\frac{\operatorname{tr}\left(\Gamma^{2}\right)}{M}=\frac{\|\Gamma\|_{F}^{2}}{M} .
$$

It follows that off diagonal elements of $\Phi \odot \Delta$ matrix are essentially product of square correlations between spreading sequences and (31). Diagonal elements are proportional to inverses of the slopes in single user communication mode.

\section{The Robust SLope Region}

Obviously, the slope region is a function of rate imbalance $\theta(A)$. Best possible case is when

$$
\begin{aligned}
R_{1}: R_{2}: \ldots: R_{K} & =\epsilon: \epsilon^{2}: \ldots: \epsilon^{K} \\
\epsilon & \rightarrow 0 .
\end{aligned}
$$

It may be verified that higher order constraints become dominated by the first order constraints and single user slopes become simultaneously achievable. For two user case this was derived in [2]. Still, if proportion between rates perhaps varies with time we would like to evaluate the following.

Definition 1 "Robust slope region" is defined as the intersection of all slope regions, taken over all possible rate proportions.

Theorem 2 Let $\Sigma$ denote diagonal matrix of inverse slopes. The robust slope region is specified by the following set of equations

$$
\begin{gathered}
\left(\mathcal{S}_{1}, \mathcal{S}_{2}, \ldots, \mathcal{S}_{K}\right): \forall A \subseteq\{1,2, \ldots, K\} \\
\left|\Sigma_{A}-\frac{L}{2} \Phi_{A} \odot \Delta_{A}\right| \geq 0 .
\end{gathered}
$$

To see that constraints (33) are sufficient note that they imply that $\Sigma_{A}-\frac{L}{2} \Phi_{A} \odot \Delta_{A}$ is positive semi-definite for every $A$, and (28) follows.

From the other side, note that (28) for every $\theta(A)$ implies that $\Sigma_{A}-\frac{L}{2} \Phi_{A} \odot \Delta_{A}$ is positive semi-definite, since its off-diagonal terms are less than zero. TDMA slope region is found in [2], and it can be shown that it is strictly contained inside the robust slope region, except in the non-fading AWGN channel, when they are the same.

Definition 2 "Robust slope" is defined as the greatest symmetric slope which belongs to the robust slope region.

The robust slope can always be granted to all users. It is not difficult to see that it is given as

$$
\mathcal{S}_{k}=\frac{2}{L} \lambda_{\max }^{-1}[\Phi \odot \Delta] .
$$

If the set of spreading codes is a design choice we may want to optimize the robust slope in order to better deal with the worst case scenario. 


\section{MANY USERS}

Here we choose to compare orthogonal and non-orthogonal strategies for many user case. set $K \gg L$, all channels i.i.d., and independent between each other and all transmission rates equal. Since $K \gg L$, we may as well chose random codes since in this case $\Phi$ is just the expected value of the squared correlation. We have that $\Phi(k, l)=1 / L$, for $k \neq l$ [4]. For large $K$, the off diagonal terms overwhelm the diagonals. It follows that

$$
\frac{1}{K^{2}} \sum_{k=1}^{K} \frac{1}{\mathcal{S}_{k}} \rightarrow \frac{1}{2}
$$

We can evaluate the bandwidth penalty due to otrhogonal codes. In orthogonal case, $L=K, \Phi$ is diagonal and we have that

$$
\frac{1}{K^{2}} \sum_{k=1}^{K} \frac{1}{\mathcal{S}_{k}} \rightarrow \frac{1}{\mathcal{S}(H)}
$$

Dividing the two quantities, we evaluate the bandwidth penalty due to orthogonalization as $2 / \mathcal{S}(H)$. For i.i.d Rayleigh fading this equals $1+M / N$. The bandwidth penalty obviously grows with increasing $M$, as we waste too many dimensions on orthogonalization. However, if $N \ll M$ an orthogonal system may be designed without sacrificing too much bandwidth.

\section{INTERFERENCE CANCELLATION}

We wish to know how interference cancellation strategies perform in the low power regime.

Theorem 3 Vertices of the "slope region" may be achieved by a matched filter linear interface, provided that it is followed by successive interference cancellation.

Equivalent statement for vertices of the capacity region is not true. It really takes a linear MMSE interface, followed by interference cancellation in order to achieve vertices of the capacity region [6]. However, if background noise is dominant over interference, then performances of the two are similar.

To demonstrate this for a simple two user case, we look at the signal to interference ratio for user 1 , after matching to the channel and the spreading sequence. Using equation (2), user 1 decoder has access to

$$
\begin{aligned}
\left(s_{1} \otimes H_{1}\right)^{\dagger} y^{\cup} & =\left(s_{1} \otimes H_{1}\right)^{\dagger}\left(s_{1} \otimes H_{1}\right) x_{1} \\
& +\left(s_{1} \otimes H_{1}\right)^{\dagger}\left[\left(s_{2} \otimes H_{2}\right) x_{2}+\eta^{\cup}\right]
\end{aligned}
$$

The first part of this equation is the "signal," and the rest is "interference." User 1 decoder treats user 2 transmission in the same manner as background noise without exploiting a possible knowledge of a user 2 code. For a fixed channel, we can evaluate that the covariance of the signal and the interference

$$
\begin{aligned}
& \operatorname{Cov}(\mathrm{S})=\left(H_{1}^{\dagger} H_{1}\right)^{2} \frac{P_{1}}{N_{1}} \\
& \operatorname{Cov}(\mathcal{I})=H_{1}^{\dagger} H_{2} H_{2}^{\dagger} H_{1}|\rho(1,2)|^{2} \frac{P_{2}}{N_{2}}+H_{1}^{\dagger} H_{1} .
\end{aligned}
$$

Furthermore, both signal and interference are Gaussian and independent from each other. Based on entropy argument, we use the following formula to find the first user rate

$$
R_{1}=E \log |\operatorname{Cov}(\mathrm{S})+\operatorname{Cov}(\mathcal{I})|-E \log |\operatorname{Cov}(\mathcal{I})| .
$$

We evaluate first and second order behaviour of this expression, using equations (11),(12) and (19). After a lot of linear algebra manipulations we obtain

$$
\begin{aligned}
& \dot{R}_{1}(0)=G_{1} \\
& \ddot{R}_{1}(0)=\frac{G_{1}^{2}}{M}\left[|\rho(1,1)|^{2} \delta(1,1)+2|\rho(1,2)|^{2} \delta(1,2) \frac{\theta_{2}}{\theta_{1}}\right] .
\end{aligned}
$$

Using (10), and normalizing per receive dimensions

$$
\frac{\theta_{1}^{2}}{\mathcal{S}_{1}}=\frac{L}{2}\left[|\rho(1,1)|^{2} \delta(1,1) \theta_{1}^{2}+2|\rho(1,2)|^{2} \delta(1,2) \theta_{1} \theta_{2}\right]
$$

Assuming perfect decision feedback, after interference cancellation the user 2 is left with the "clean" channel,

$$
\frac{\theta_{2}^{2}}{\mathcal{S}_{2}}=\frac{L}{2}|\rho(2,2)|^{2} \delta(2,2) \theta_{2}^{2}
$$

Adding up the two equations we see that the joint slope constraint is satisfied.

\section{CONCLUSION}

The slope region for multiple users with spreading codes and multiple antennas is described by a simple quadratic expression. This region is essentially a function of the relative proportion between the users rates and the channel statistics. The robust slope region is inside every other slope region and it only depends on channel statistics. The "robust slope" can always be granted to all users, regardless of the relative proportion between the rates. Matched filter linear interface suffices to achieve vertices of the slope region, provided that we use interference cancellation.

\section{REFERENCES}

[1] S. Verdu, "Spectral Efficiency in the Wideband Regime" IEEE Transactions on Information Theory, vol. 48, pp.1319-1343, June 2002.

[2] G. Caire, D. Tunetti and S. Verdu "Suboptimality of TDMA in the Low Power Regime" submitted to IEEE Transactions on Information Theory.

[3] S. Verdu, G. Caire and D. Tunetti "Is TDMA Optimal in the Low Power Regime?" Proc. of ISIT 2002, pp 193, July 2002.

[4] S. Verdu, "Spectral Efficiency of CDMA with Random Spreading" IEEE Transactions on Information Theory, vol. 45, pp.622-640, March 1999.

[5] S. Shamai and S. Verdu, "The Impact of Frequency-Flat Fading on the Spectral Efficiency of CDMA" IEEE Transactions on Information Theory, vol. 47, pp.1302-1327, May 2001.

[6] M. K. Varanasi and T. Guess "Optimum Decision Feedback Multiuser Equalization with Succesive Decoding Achieves the Total Capacity of the Gaussian Multiple-Access Channel" in Proc. Asilomar Conf., Pacific Grove, CA, Nov. 1997.

[7] A. Graham, "Kronecker Products and Matrix Calculus with Applications" Halsted Press, John Willey and Sons, NY, 1981. 